\title{
Urgences
}

\section{Les nouvelles de Grandbois comme objet transitionnel}

\section{Nicole Deschamps}

Numéro 24, juillet 1989

Le manuscrit sous l'angle

URI : https://id.erudit.org/iderudit/025528ar

DOI : https://doi.org/10.7202/025528ar

Aller au sommaire du numéro

Éditeur(s)

Urgences

ISSN

0226-9554 (imprimé)

1927-3924 (numérique)

Découvrir la revue

Citer cet article

Deschamps, N. (1989). Les nouvelles de Grandbois comme objet transitionnel. Urgences, (24), 17-23. https://doi.org/10.7202/025528ar d'utilisation que vous pouvez consulter en ligne.

https://apropos.erudit.org/fr/usagers/politique-dutilisation/ 


\title{
Les nouvelles de Grandbois comme objet transitionnel
}

\author{
Nicole Deschamps, \\ Université de Montréal
}

À présent que Chantal Bouchard et moi-même achevons le travail d'édition critique des nouvelles d'Alain Grandbois, l'idée nous est venue de prendre un peu de recul pour nous poser la question de leur signification dans l'ensemble de l'œuvre sur laquelle nous travaillons depuis quelques années. Alors que ma collègue de l'université McGill présentera l'aspect analytique de notre recherche en exposant les problèmes particuliers posés par l'édition critique de "Julius", à la fois la plus autobiographique et la plus complexe des nouvelles d'un point de vue technique, j'en aborderai l'aspect synthétique en proposant l'esquisse d'une lecture d'ensemble des nouvelles, celles que nous publierons et celles que nous n'avons pas retenues. L'idée sur laquelle s'appuie mon hypothèse est celle du manuscrit inachevé comme objet transitionnel, thème qui a déjà été exploré d'un point de vue théorique par Christian Béthune dans un article de 1972: “Qu'est-ce qu'un brouillon? ou le brouillon, objet transitionnel" et qui a été repris en diverses ramifications et illustrations dans le Manuscrit inachevé, recueil d'études publiées par le CNRS en 1986.1

L'œuvre et la réputation d'Alain Grandbois reposent évidemment sur sa poésie qui ne risque pas d'être oubliée. S'il existe une voix propre à Grandbois, c'est d'abord là qu'elle parle, et Jacques Brault l'a déjà éloquemment mise en valeur. ${ }^{2}$ ॥ y a également, chez ce poète de la modernité naissante au Québec, un écrivain explorateur d'autres formes littéraires qui a continué à se chercher sans jamais se fixer vraiment. On le connaît par les pudiques souvenirs et récits de voyage qu'il a

1. L'article de Christian Béthune a paru dans Pour l'Objet, Revue d'esthétique, nos 3-4, 1979, p. 43-52. Du recueil de communications sur le Manuscrit inachevé, soulignons celle de Jean-Louis Lebrave, "L'écriture interrompue: quelques problèmes théoriques", p. 127-163. Paris, Éditions du CNRS, 1986, 155 p.

2 Jacques Brault, Alain Grandbois, coll. «Poètes d'aujourd'hui», Montréal, l'Hexagone et Paris, Segners, 1968, 190 p. 
publiés et diffusés à la radio, ainsi que par les nouvelles d'Avant le chaos. ${ }^{3}$ II est présent dans ses brouillons, particulièrement dans les ébauches de ses nouvelles, récits ou romans. Nous en esquisserons plus loin le contenu. Dans une certaine mesure, il ne serait peut-être pas faux d'assimiler ces textes délaissés par l'auteur, mais jamais traités en purs déchets, à l'ensemble des ouvrages publiés en prose.

Pour donner une idée de la quantité des documents, citons les chiffres établis par Danielle Rompré 4 qui avait présenté, dès 1977, un premier classement des manuscrits: 9104 feuillets manuscrits, 599 feuillets dactylographiés, 1086 feuillets dactylographiés au carbone, ce qui fait en tout 10789 feuillets. Le nombre exact des nouvelles n'est pas facile à fixer puisque plusieurs textes demeurent inclassables. Nous en avons repéré une quarantaine.

Cette partie importante de l'œuvre de Grandbois est marquée par une recherche autobiographique qui s'avoue plus ou moins ouvertement. Ainsi, les voyages racontés sous le mode du récit pittoresque dans Visages du monde se retrouvent, à peine transposés, dans les paysages des nouvelles au décor exotique. Quant aux deux récits d'aventures qui empruntent autant à la fiction qu'à la documentation historique: Né à Québec et Les voyages de Marco Polo, il serait facile d'y lire, au delà des conquêtes de l'espace, un voyage intérieur et d'y reconnaître, dans le personnage des héros, quelques-uns des masques de l'auteur.

Mais c'est surtout dans les nouvelles que ressortira l'évidence de la fonction de ces écrits, catalyseurs de la poésie. Commençons par situer dans la vie de Grandbois les premières traces de son activité de nouvelliste: elles correspondent à son

3 Dans l'ordre chronologique de leur parution, Né à Québec, Louis Jolliet, Récit, coll. du «Nénuphar», Montréal, Fides, 1948, 207 p., accompagné d'une préface de Luc Lacoursière, p. 9-10; Les voyages de Marco-Polo, coll. du aNénuphar», Montréal, Fides, 1969, 174 p. accompagné d'une préface de Jacques Blais, p. 7-11; Avant le chaos, coll. al'Arbre», Montréal, HMH, 1964, 276 p. (en cours de texte AC suivi de la page); Visages du monde. Images et souvenirs de l'entre-deux guerres, coll. "Reconnaissances", Montréal, HMH, 1971, 378 p., accompagné d'une préface de Léopold Leblanc, p. 11-16.

4 Fonds Alain Grandbois, inventaire dressé par Danielle Rompré, Montréal, Bibliothèque nationale du Québec, 1977, 106 p. 
retour précipité au Canada en 1939. C'est le moment où seront perdus les manuscrits de jeunesse. Rappelons que les quatre premières nouvelles d'Avant le chaos ont paru d'abord en 1942, à Montréal, dans des revues (Revue moderne, Revue populaire) puis en 1945, aux éditions Modernes. Un second recueil a paru en 1964, chez HMH, ajoutant quatre nouvelles à la réédition des premières. À remarquer que si les premiers poèmes de Grandbois ont été publiés d'abord à Hankeou, en Chine, aux antipodes de son milieu d'origine, les nouvelles paraissent sous le signe du retour au pays, retour qui a été vécu comme un enlisement. Avant le chaos présuppose une catastrophe qui n'est pas seulement celle de la guerre 39-45 dont parle l'avant-propos, mais une crise personnelle de l'auteur: faillite d'une vie de liberté marquée par l'insouciance matérielle, période de voyages et d'aventures vécue de 1920 à 1939, ce qui correspond à l'âge de Grandbois né en 1900, jeunesse qui paraît désormais d'autant plus fabuleuse qu'elle est irrémédiablement finie.

Par la suite du fatidique retour au Canada en 1939, Grandbois va maintenir un lien avec son activité de nouvelliste, sans pourtant ajouter substantiellement à la réédition d'Avant le chaos, en 1964. D'autres ébauches s'accumuleront sans pourtant franchir le seuil d'Avant le chaos: elles demeurent champ d'exploration, exutoire autobiographique, laboratoire d'essais et, à ces titres, elles ont un róle vital.

Empreintes de nostalgie, les nouvelles bien connues d'Avant le chaos mettent en scène un narrateur cultivé, riche, jeune et beau, d'origine canadienne; il est poète et parfois romancier, comme dans "Grégor" où il écrit Samiah (roman qui existe bel et bien dans les archives mais que ni le narrateur ni l'auteur n'achèveront ${ }^{5}$ ); il est amoureux sans être heureux en amour; il voyage en Europe, en Asie, en Afrique et il passe beaucoup de temps dans les bars. II se projette également dans les personnages secondaires, par exemple, Christian, le mari de Tania dans la nouvelle du même nom: "c'était un jeune homme, dans la trentaine, extrêmement courtois, de manières un peu

5 Samiah ou l'Épisode ingénu, également sous les titres de Amia, Les désirs de l'incertitude et autres, manuscrit de 143 feuillets, Fonds Grandbois, BNQ, boite 2, chemise 34B. Des fragments de Samiah ou l'épisode ingénue ont paru dans Délivrance du jour et autres inédits, avec des dessins de l'auteur, Montréal, Éditions du Sentier, 1980, p. 33-41. 
distantes" (AC, p.67). Quelques inédits exploitent cette veine qui est celle de la plupart des nouvelles publiées, à l'exception du "Noël de Jéróme" et de "lls étaient deux commandos".

Mais les ébauches les plus intéressantes sont celles qui tentent de décrire la société québécoise. On pourrait les regrouper sous deux thèmes:

1) une série autobiographique qui met en scène un jeune homme étudiant à Québec, observateur impitoyable de la bourgeoisie où il évolue. Par exemple Le discours dangereux qui raconte une ballade en voiture durant laquelle le narrateur écoute parler un médecin réputé dont l'univers mental est celui des voitures puissantes qu'il n'ose même pas faire rouler trop vite. Parmi ces textes, des ébauches de romans de mœurs qui n'arrivent pas à dépasser la forme du tableau: Charlie Tessier, Gilberte...6

2) une série plus surprenante de nouvelles scabreuses dans lesquelles le personnage du narrateur, plus ou moins identique à l'auteur, est éliminé: sordides histoires de meurtres, de vols, de viols, de tabassages par la police, racontées soit à la première personne (Un raté), soit à la façon d'un reportage à la troisième personne (Un grand homme). ${ }^{7}$ Soulignons l'intérêt linguistique de ces écrits. Dans une communication récente, Chantal Bouchard a fait une étude comparative des dialogues chez Grandbois en montrant l'extrême difficulté où l'auteur d'Avant le chaos s'est trouvé pour faire parler ses compatriotes avec naturel. ${ }^{8}$

Sans vouloir introduire ici d'inutiles clivages entre les nouvelles que Grandbois a choisi d'éditer et les autres, entre son activité de poète et celle de prosateur, on pourrait voir une part de son œuvre comme l'interminable brouillon (l'indispensable

6 Le discours dangereux, manuscrit de 5 feuillets, Fonds Grandbois, BNQ, boîte 3, chemise 5; Charlie Tessier, manuscrit de 10 feuillets, Fonds Grandbois, BNQ, boite 3, chemise 5; Gilberte, manuscrit de 35 feuillets, Fonds Grandbois, BNQ, boite 3, chemise 6.

7 Un raté, manuscrit de 3 feuillets et Un grand homme, manuscrit de 4 feuillets, Fonds Grandbois, BNQ, boîte 3, chemise 16. A côté de ces ébauches de quelques pages, on en trouve de plus développées comme Un filet de sang, manuscrit de 87 pages, loc. cit.

8 Les nouvelles d'Alain Grandbois: donner la parole aux personnages», communication présentée à Catania (Italie) en 1988. 
placenta?) qui assure la survie de la production poétique. Sur une autre scène, on pourrait voir la fonction du brouillon chez Grandbois comme la bouée de sauvetage de son narcissisme, narcissisme sans lequel aucun destin humain n'est concevable, à plus forte raison celui d'écrivain.

Au risque de paraître scolaire, j'éluciderai maintenant brièvement les notions pertinentes à l'objet transitionnel. On sait que le concept d'objet transitionnel a été introduit dans la théorie psychanalytique par Winnicott, psychanalyste anglais spécialisé dans le soin des enfants. En bref, c'est l'objet, par exemple un ours en peluche ou un coin de couverture, que l'enfant va substituer au sein de sa mère dans la période de passage qui le mènera, par l'épreuve de la réalité, à une véritable relation d'objet. Dans l'expérience de l'enfant, il se situe à mi-chemin entre le subjectif et l'objectif et il appartient, selon Winnicott, au domaine de l'illusion (mais non de l'hallucination). "L'objet transitionnel et le phénomène transitionnel, dit-il, apportent, dès le départ à tout être humain, quelque chose qui restera toujours important pour lui, à savoir un champ neutre d'expérimentation qui ne sera pas contesté". 9

Du point de vue qui nous intéresse, le brouillon s'impose donc, suivant l'expression de Christian Béthune, "comme moyen terme indispensable entre le sujet et son œuvre". II précise que cette médiation n'est ni «linéaire, ni même dialectique" et qu'elle "se déploie dans l'espace vacillant d'un mouvement pendulaire qui détermine la spécificité de son être et de son sens". À l'expérience du brouillon comme à celle de l'objet transitionnel, correspondraient quatre critères ainsi résumés par Béthune: «1) L'objet transitionnel représente pour le sujet le premier objet effectivement perçu comme non moi. 2) II se manifeste essentiellement comme limite, dans la mesure où le sujet hésite à le situer nettement à l'intérieur ou à l'extérieur. 3) II délimite pour le sujet un champ neutre d'expérience. 4) II symbolise enfin l'union entre deux termes". 10

9 Cité par J. Laplanche et J.B. Pontalis dans leur Vocabulaire de la psychanalyse, Paris, PUF, 1967, p. 296. Soulignons qu'en micropsychanalyse, Silvio Fanti insiste sur la «pérennité sous-jacenten de l'attachement à l'objet transitionnel. Cf. le Dictionnaire pratique de la psychanalyse et de la micropsychanalyse, Paris, Buchet/Chastel, 1983, p. 218.

10 Christian Béthune, op. cit., p. 44-45. 
Sans élaborer sur chacun de ces critères, rappelons que "l'art et la technique assument initialement le fantasme d'un désir d'omnipotence"11, ce qui met d'emblée de connivence l'auteur et le technicien des variantes. Dès qu'il sera à l'ouvrage, tout artisan devra reconnaître ses limites puisqu'il demeure assujetti à toutes sortes de lois contraignantes. De ce fait, le manuscrit inachevé serait le lieu idéal, à la fois conflictuel et supportable, entre principe de plaisir et principe de réalité. L'exemple le plus éclatant qu'on pourrait donner d'un tel phénomène serait sans doute la Recherche de Proust.

Quant à notre travail de repérage à travers les brouillons, ou de relevé des variantes d'une œuvre déjà constituée, il participe à l'ambiguiité de la situation de l'auteur par rapport à son œuvre. L'auteur n'ayant su trancher entre ce qu'il voulait conserver et ce qu'il souhaitait jeter, comment d'autres sauraient-ils mieux le faire à sa place?

Grandbois nouvelliste et, par la, mystérieux autobiographe, reste à découvrir. Avant le chaos n'a peut-être pas eu la diffusion qu'il méritait car ce livre dit plus que son pittoresque de surface. Pourtant, comme l'a récemment fait remarquer JeanPierre Boucher à propos de la forme éclatée du recueil: «peu d'œuvres québécoises touchent autant notre sensibilité actuelle". 12 Est-ce bien le poète des îles de la nuit qui trouve dans sa prose ces accents beckettiens? "Qui sommes-nous, petits insectes humains, nés aux hasards des copulations, choisis entre 100,000 spermatozoïdes, dans un seul acte de copulation entre un mâle et une femelle, qui sommesnous?..., 13

L'essai de mettre en forme des narrations va donc jouer chez Grandbois un rôle vital pour lui-même et pour son œuvre, en lui permettant à la fois de restaurer son narcissisme et d'approfondir la connaissance de lui-même dans le désarroi d'une conscience contemporaine. C'est sans doute pourquoi il ne se détachera jamais de cette activité qu'il prolongera toute sa vie, sans pourtant accoucher d'un véritable roman ni en finir

\section{Loc. cit., p. 45.}

12 "Fragmentation et unité dans Avant le chaos" Ecrits du Canada français, no 60, Montréal, HMH, 1987, p. 156.

13 Manuscrit sans titre de 8 feuillets, Fonds Grandbois, BNQ, boite 3, chemise 1. 
vraiment avec l'idée d'en écrire un. Les nouvelles demeurent en grand nombre inachevées. Activité marginale à celle de l'écriture des poèmes, mais pourtant essentielle. Activité évidemment autobiographique, mais également très masquée. Cela tient au fait que les nouvelles sont presque toutes composées de plusieurs histoires qui sautent de l'une à l'autre; il devient alors impossible d'en achever aucune. Les nouvelles les plus achevées demeurent donc un brouillon infini, sorte d'objet transitionnel que Grandbois va traîner avec lui toute sa vie après le chaos, ce chaos qui pourrait s'entendre aussi comme le lieu des origines introuvables, insondables.

Alain Grandbois, pourtant enraciné dans un milieu familial et social relativement favorable à son épanouissement, acclamé et respecté par ses contemporains, n'a jamais pu s'identifier tout à fait à son personnage. Et il n'a jamais pu s'installer nulle part. Attendant le train, l'avion, le bateau ("Quand j'étais à Rome, écrit-il dans un fragment inédit, je voulais être à Pékin, quand j'étais à Port-Cros, je regrettais Paris, ma vie sentimentale était aussi inconséquente..."14), en voyage donc, ou bien assis dans un bar au Cambodge, à Québec ou à Madrid, il est là, comme tant de ses contemporains, en attendant Godot... II voudrait certes s'accrocher à ses souvenirs en les racontant, mais il n'y arrive pas. Ni à y croire, ni à les écrire. Pourtant, il témoigne certainement de ce qui nous touche dans la perception de la continuité/discontinuité qui crée un destin - pour lui, d'être écrivain, à travers tous les hasards de sa vie déterminée par ses rêves. Les voyages d'Alain Grandbois sont aussi une métaphore d'une exploration de lui-même plus radicale, qu'il a faite par l'écriture.

14 Manuscrit sans titre de 14 feuillets, Fonds Grandbois, BNQ, boîte 3 , chemise 1, p. 8. 